\title{
Derivati in -igno. Tra lingua, dialetto e italiano regionale letterario
}

\author{
Salvatore C. Trovato \\ Università degli Studi di Catania \\ salvtrov@libero.it
}

\begin{abstract}
L'autore mette a confronto il suff. it. -ign|o con il corrispondente siciliano e calabrese -ign $\mid u$, rilevando, sulla base di tutti gli esempi disponibili, la moderata produttività del suffisso italiano, rispetto alla maggiore produttività del corrispondente suffisso del dialetto. La maggiore produttività di quest'ultimo si ripercuote sull'italiano letterario di due grandi scrittori espressionisti: il siciliano Stefano D’Arrigo e il calabrese Giuseppe Occhiato. Di questi autori vengono ricordate tutte le forme in -ign|o presenti nei loro romanzi e presentate alcune schede di parole nuove alla lessicografia italiana.

Parole chiave: derivazione, alterazione, lingua, dialetto, italiano letterario.

\section{Abstract}

The author compares the Italian suffix -ign|o with the equivalent Sicilian and Calabrian -ign $\mid u$, pointing out, on the basis of all the existing examples, the modest productivity of the Italian suffix, in comparison with the greater productivity of the equivalent suffix in the dialect. The superior productivity of the dialect influences the Italian literary production of two great expressionist writers: the Sicilian Stefano D'Arrigo and the Calabrian Giuseppe Occhiato. All the forms with -ign|o used by those authors in their novels are quoted as well as some examples of words new to the Italian lexicography.
\end{abstract}

Key words: derivation, language, dialect, literary Italian.

\section{Motivazione di un'indagine}

Il presente contributo nasce dall'esigenza di capire e motivare la presenza, in Horcynus Orca di Stefano D'Arrigo (da ora in poi sempre $H O)^{1}$ e in Oga Magoga di Giuseppe Occhiato (da ora in poi sempre $O M$ ), ${ }^{2}$ dei derivati in -igno, suffisso che, com'è noto, non è particolarmente produttivo in italiano, ma che

2. Giuseppe Occhiato, Oga Magoga, Cosenza: Editoriale Progetto, 2000. 
conta 11 derivati (con 55 occorrenze) in Stefano D'Arrigo e 28 derivati (con 237 occorrenze) in Giuseppe Occhiato. Entrambi gli scrittori -il noto D'Arrigo e il meno noto (ma non meno importante) Occhiato- si situano nell'ambito dell'espressionismo linguistico. Essi, infatti, hanno attinto a piene mani la loro lingua - e entrambi in maniera originalissima ${ }^{3}$ alle fonti del dialetto. Il dialetto in entrambi i casi, per usare un'espressione cara a D'Arrigo, è quello dello "Scill'e Cariddi», la zona dello Stretto, e cioè il siciliano dell'area messinese ionica per D’Arrigo e il calabrese meridionale della zona di Mileto per Giuseppe Occhiato.

Prima di entrare nel merito dei derivati in -igno nei nostri due scrittori, credo opportuno esaminare la situazione di quel suffisso: a) in italiano, sulla base dei dati contenuti in DM; ${ }^{4}$ b) in siciliano, sulla base del Vocabolario siciliano inverso (Trovato-Valenti in stampa), e in calabrese, sulla base dei pochi dati contenuti nel NDDC di Rohlfs 5 e su alcuni controlli eseguiti personalmente nella Calabria meridionale estrema e nel Messinese.

\section{Il suffisso -igno in italiano}

Il suffisso -ign $\mid o$ - utilizzato a formare aggettivi di relazione, quando la base è costituita da un nome, o aggettivi alterati, quando la base è anch'essa un aggettivo- in italiano è moderatamente produttivo. DM CD-Rom ricorda poco più di quaranta derivati. Li ricordo, distinguendo:

a) derivati a base nominale (qui inclusi alcuni pochi che hanno come base un confisso) $(\mathrm{N} \rightarrow \mathrm{A})$ :

benigno $\leftarrow$ bene, boschigno $\leftarrow$ bosco, cancerigno $\leftarrow$ [cancer- $]^{6}$ conf. + suff., caprigno $\leftarrow$ capra, ciprigno $\leftarrow$ Cipro, ferigno e inferigno $\leftarrow$ (in) + fera + suff., ferrigno $\leftarrow$ ferro, ferrugigno $\leftarrow[\text { ferrug- }]^{\top}$ conf. + suff., lupigno $\leftarrow$ lupo, maligno $\leftarrow$ male, nervigno $\leftarrow$ nervo, olivigno e ulivigno $\leftarrow$ olivolu-, petrigno $\leftarrow$ pietra, salcigno $\leftarrow$ salce, saligno $\leftarrow$ sale e saligno $\leftarrow$ sal( $(i c) e$, sanguigno $\leftarrow$ sangue, segaligno $\leftarrow$ segale, selcigno $\leftarrow$ selce, serpigno $\leftarrow$ serpe, sorcigno $\leftarrow$ sorcio, sterpigno $\leftarrow$ sterpo, terrigno $\leftarrow$ terra, vespigno $\leftarrow$ vespa, vetrigno $\leftarrow$ vetro, volpigno $\leftarrow$ volpe;

b) derivati —in realtà «alterati» ${ }^{8}$ — a base aggettivale $(\mathrm{A} \rightarrow \mathrm{A})$ :

3. Per D'Arrigo rimando particolarmente a Salvatore TrOVATO, «La formazione della parole in Horcynus Orca di Stefano D’Arrigo. Tra regionalità e creatività», Quaderni di Semantica, XXVIII, 2007, p. 41-8, e per Occhiato a A. PIROMALlI, "Giuseppe Occhiato narratore epico-popolare», Letteratura \& Società, IV/2, 2002, p. 35-50.

4. $\mathrm{DM}=\mathrm{T}$. De MAUro (a cura di), Il dizionario della lingua italiana, Torino: Paravia 2000.

5. $\mathrm{NDDC}=\mathrm{G}$. RoHLfs, Nuovo Dizionario dialettale della Calabria, Ravenna: Longo 1977

6. In DM è data l'etimologia diacronica: «der. del lat. cancer "cancro" con -igno».

7. DM dà l'etimologia diacronica: «lat. ferruğ̌nĕu $(m) »$.

8. Non è inutile ricordare che, sul piano formale, come per tutti i suffissi valutativi, anche per in quelli in -igno, che riporto in (b), la categoria sintattica di base non cambia. 
acrignolagri- $\leftarrow$ acrel agro, asprigno $\leftarrow$ aspro, azzurrigno $\leftarrow$ azzurro, crespigno $\leftarrow$ crespo, dolcigno $\leftarrow$ dolce, fortigno $\leftarrow$ forte, gialligno $\leftarrow$ giallo, lividigno $\leftarrow$ livido, nerigno $\leftarrow$ nero, rossigno $\leftarrow$ rosso, smortigno $\leftarrow$ smorto, verdigno $\leftarrow$ verde;

c) derivati del tipo:

padrignolpatri- e madrignalmatri- e privigno (femm. privigna) il cui movimento derivazionale è $\mathrm{N} \rightarrow \mathrm{N}$. 9

Del tutto oscuro ${ }^{10}$ è, in sincronia, privignol-a «figliastro,-a». L'analisi etimologica, però, permette di ravvisarvi un composto, formato da prīvus/-a agg.e dal tema *gen- (DEI IV 3085, DM CD-Rom). Sulla forma femm. privigna, successivamente, si è formato matrigna, forma attestata nelle glosse (DEI III 2308) e, di rimbalzo, anche il non documentato *patrignus (DEI IV 2805), cui va riportato l'it. patrigno. Ora è chiaro che ciò è avvenuto per desemantizzazione e conseguente grammaticalizzazione di -igno, fenomeno che, nel romanzo di Occhiato, come vedremo, ha continuato ad essere produttivo dando luogo a fratigno "fratellastro». E -igno, in questa ristrettissima classe di nomi parentelari, ha assunto valore valutativo, acquistando lo stesso valore che nei dialetti ha -ast $(r) \mid u$, come nei sicc. patrastru e parrastru; mammastra, marrastra, parrastra; fratastru; surastra e nei call. (NDDC) parrastrulparrastu, sirastu, tatast « per "patrigno», mammastra, matrastra, parrasta, fratastru, sorastra ecc.

Sulla base della documentazione esposta, non si può dire che -igno sia particolarmente produttivo, se si paragona ad es., particolarmente per quel che riguarda le funzioni in (a) e (b), a -ino (con circa 1700 forme registrate in DM CD-Rom), oppure a -oso (con 1102 derivati registrati nello stesso CD-Rom). ${ }^{11}$ Wandruszka, infatti, lo dà come suffisso "marginalmente produttivo», ${ }^{12}$ e la Merlini Barbaresi — che studia il suffisso nella sua funzione di alterativo- lo definisce "poco produttivo» o addirittura «inconsueto» ma solo a proposito del darrighiano scaltrigno. ${ }^{14}$

9. Analogo processo derivativo $(\mathrm{N} \rightarrow \mathrm{N})$ si osserva nell'obsoleto colmigno "comignolo», in cui, in sincronia, è facile ravvisarvi la base colmo «linea di colmo», ma per il quale DM CDRom propone l'analisi diacronica: < lat. *ulmŭnĕu $(m)$, der. di culmen, -inis «sommità».

10. Non prendo in considerazione nomi come macigno, in sincronia oscuro nei suoi componenti, e le voci regionali (toscane) raffrigno "cucitura sommaria con punti radi e disuguali, ecc.», rinfrigno «rammendo mal fatto, ecc.» e fuffigno «ingarbugliamento dei fili di una matassa o di un tessuto".

11. Ma cfr. anche Salvatore C. SGROI, "(Im)produttività dei suffissi -oso, -ioso, -uoso e problemi di etimologia sincronica e diacronica», in Quaderni di Semantica, XXI, 2000, p. 265-319, e, per la presenza di esso in Horcynus Orca, Trovato op. cit.

12. Cfr. U. Wandruszka, "Aggettivi denominali», in M. Grossmann, F. Rainer (a cura di), La formazione delle parole in italiano, Tübingen: Niemeyer, 2004, p. 382-402, (p. 397).

13. L. Merlini Barbaresi, "Aggettivi deaggettivali», in M. Grossmann, F. Rainer (a cura di), La formazione delle parole in italiano, Tübingen: Niemeyer, 2004, p. 444-450 (p. 445, 447: ma poco prima (p. 446) lo aveva annoverato tra i suffissi «attenuativi produttivi»).

14. L. Merlini Barbaresi, «Alterazione», Ibid., p. 264-292. 
Nei dialetti, invece - mi riferisco particolarmente a quello siciliano e al calabrese meridionale, che del primo per tanti aspetti può considerarsi un'appendice- il suffisso è particolarmente produttivo e ancora vivo. Nel VS si trovano poco meno di 200 aggettivi di relazione e/o alterati in -ign $\mid u .{ }^{15}$

\section{Il suffisso -ignu nel siciliano e nel calabrese}

Nel siciliano il suffisso è particolarmente produttivo. Sulla base del Vocabolario siciliano inverso (Trovato-Valenti in stampa), del NDDC di Rohlfs e di un supplemento di indagini condotte nella Calabria reggina ${ }^{16} \mathrm{e}$ a Messina è possibile elencare 109 forme (varianti incluse) in cui -ign|u si lega a una base nominale ed ha, ovviamente, valore derivativo:

a) $\mathrm{N} \rightarrow \mathrm{A}$ :

acquignu «acquoso» $\leftarrow$ acqua, arbirignu «di legname duro che si sgretola con facilità" $\leftarrow$ àrbiru, (a)gghjarignu "ghiaioso" $\leftarrow$ (a)gghjara, bbicchignu "che odora di becco» ecc. $\leftarrow$ bbeccu, bbinignul bbirignu «benigno» $\leftarrow$ bbeni ecc., bbuschignulvuschignu «boschereccio» $\leftarrow$ bboscu ecc., (petra) bbucalacigna «calcare riccamente fossilifero» $\leftarrow$ bbucalaci «chiocciola», (vista) bbuigna "vista corta» e â üigna loc. avv. «alla maniera dei buoi, di equino che scalcitra lateralmente» $\leftarrow$ bboi/uoi «bue», canapignu «simile a canapa» $\leftarrow$ canapa, cannavignu «di canapa, canapino» $\leftarrow$ cànnavu «canapa», cantarignu ${ }^{17}$ (solo cal.: NDDC) $\leftarrow$ cantaru, carcarazzignu "ciarliero" $\leftarrow$ carcarazza "gazza", carnignu «carnicino» $\leftarrow$ carni, carusignu «che compie azioni puerili» $\leftarrow$ carusu, cavaddignu "cavallino, che è proprio del cavallo» $\leftarrow$ cavaddu, chjummignu «che ha il colore o la qualità del piombo" $\leftarrow$ chjummu, çianchignulhianchignu/scianchignu «di persona o cosa che sta o si muove inclinata su di un fianco» $\leftarrow$ çiancul hiancu, cicirignu "che ha il colore del cece» $\leftarrow$ cìciru, cirividdignul cirviddignu «sventato, leggero» $\leftarrow$ ciriveddulcirveddu, citrignu (anche cal.: NDDC)/tritrignul tricignu «fitto, serrato come la polpa del cedro" $\leftarrow$ citru ecc., ciuccignu (solo cal.) $\leftarrow$ ciucciu, cozzignu «ostinato, dalla dura cervice» (solo cal.) $\leftarrow$ cozzu "nuca», crapignu (anche cal.) «caprino; dai capelli ispidi» $\leftarrow$ crapa, crastignu «incivile, sgarbato» $\leftarrow$ crastu «montone», critignu «argilloso» $\leftarrow$ crita, cucchignu «mezzo scemo» $\leftarrow$ cucca "civetta», ${ }^{18}$ culignu «di naso molto sensibile agli odori» $\leftarrow$ culu, (petra) cutigna "pietra dura, liscia e impermeabile» $\leftarrow$ cuti "ciottolo; cote», cuttunignu "cotonoso" $\leftarrow$ cuttuni, (nucidda) favigna "varietà di nocciola» $\leftarrow$ fava, firrignulferrignu (cal.)/ «simile al ferro, per colore, durezza ecc.»/(petra) furrigna "pietra lavica» $\leftarrow$ ferru, frummintignu «biondo» $\leftarrow$ frummentu, gattignu (anche cal.: NDDC)/iattignu "gattesco" $\leftarrow$ gattuliattu, lattignu «di bambino che presenta sul capo croste di lattime» $\leftarrow$ latti, liprignu «leporino (del labbro)» $\leftarrow$ lepri, lupignu «lupesco» $\leftarrow$ lupu, malignulmarignu

15. Per il calabrese non ho dati precisi. IL NDDC di Rohlfs ne registra solo alcuni e non ha approntato un indice inverso.

16. Le forme in questo caso sono indicate con «cal.», «anche cal.» o «solo cal.».

17. Solo nella loc. sarma cantarigna "carico molto pesante» (NDDC).

18. Si ricordi, per la semantica, l'it. allocco. 
«maligno» $\leftarrow$ mali/mari, manchignu «rivolto a tramontana» $\leftarrow$ manca «tramontana», margignu "paludoso, acquitrinoso» $\leftarrow$ màrgiu «acquitrino», marmurignu "marmoreo" $\leftarrow$ màrmuru, mastrignu "da maestro" $\leftarrow$ mastru, mischignu «brizzolato, riferito ai capelli» $\leftarrow$ misca «miscuglio, miscela», mulignu «da mulo» $\leftarrow$ mulu, muntignu «che ha la fronte sporgente e ampia» $\leftarrow$ munti «monte», muscatiddignu «di fichidindia dalla polpa bianca e zuccherina» $\leftarrow$ muscateddu «moscatello», nirvignu/ nirbignu «robusto, vigoroso» $\leftarrow$ nervu ecc., (varva) nzitigna "barba ispida» $\leftarrow$ nzita «setola», passarignu «simile al passero» $\leftarrow$ pàssaru, picurignu «simile a pecora» $\leftarrow$ pècura, pircialignu «di sasso non serrato, ma ghiaioso, quasi un mucchio di breccia» $\leftarrow$ pirciali «breccia»,pircupignu «di una varietà di susina simile alle albicocche» $\leftarrow$ pircopu «albicocca», pirnicignu «che ha il colore della pernice» $\leftarrow$ pirnici, pischignu «(di) $)^{19}$ luogo molto umido e fangoso per acqua o altro liquido" $\leftarrow$ pisca "fango, melma" o piscu «fanghiglia», prumunignu «soffice, spugnoso come il polmone» $\leftarrow$ prumuni "polmone», punentignulpunintignu «di vento di ponente» $\leftarrow$ punenti, purcignu «a somiglianza del porco» $\leftarrow$ porcu, purpurignu «del colore della porpora» $\leftarrow$ pùrpura, salignu «salmastro» $\leftarrow$ sali, sanguignu (anche cal.) Isanquignu/sanghignulsagnignu "sanguigno» $\leftarrow$ sangu/sancu ecc., sapunignu «saponaceo» $\leftarrow$ sapuni, sarcignu "amaro, di cattivo sapore» $\leftarrow{ }^{*}$ sarci $\leftarrow$ sàlici «salice», savuchignu "lentigginoso, dalla pelle pigmentata» $\leftarrow$ sàvucu «sambuco» e estens. e coll. «lentiggini», sbirrignu per lo più nel pl. «indagatori, degli occhi» $\leftarrow$ sbir$r u$ «sbirro», scattrignu ${ }^{20} \leftarrow$ scattru «scaltro», scavignu "piuttosto bruno, di equino dal mantello scuro, tendente al nero» $\leftarrow$ scavu «morello, equino dal mantello nero", scicchignu «asinino" $\leftarrow$ sceccu «asino», (vuci) schirdigna "voce squillante e molto acuta" $\leftarrow$ schirdu "grido acuto", scirrignu «litigioso" $\leftarrow$ scerra «lite», sgarrignu «strabico; torvo; malandrino, brigante» $\leftarrow$ sgarru, ${ }^{21}$ spirdignu «spiritato (degli occhi)» $\leftarrow$ spirdu «spirito, fantasma», surcignulsuricignulsurgignu/suggignu «sorcino, dal color cenere» $\leftarrow$ surci «topo», surfarignu (anche cal.: NDDC) «causato dallo zolfo o del colore dello zolfo»/(acqua) suffrigna "sorgente solforosa» $\leftarrow$ sùrfaru, tabbacchignu "del colore simile a quello del tabacco» $\leftarrow$ tabbaccu, taurignu "taurino" $\leftarrow$ tàuru, trucchignu (solo cal.) $\leftarrow$ truccu «trucco", (petra) tufigna "tufo, roccia derivante dalla cementazione di frammenti di origine vulcanica» $\leftarrow$ tufu «id.», tuppignu «del campanaccio che emette un suono squillante» $\leftarrow$ tuppi onomat., turbignu «calcareo, di terreno» $\leftarrow$ turba «terreno calcareo», (rracina) ulivigna "varietà di uva» $\leftarrow$ uliva, ursignulussignu «scorbutico, scontroso $\leftarrow$ ursu ecc., vacchignu «della vacca; di cavallo dal mantello macchiato di bianco, ecc.» $\leftarrow$ vacca, â vastasigna «in maniera indecente» $\leftarrow$ vastasu "persona incivile e maleducata», vitrignu "fragile come il vetro, ecc.» $\leftarrow$ vitru, vurpignulvuppignu/urpignu/(anche cal.) gurpignu «volpino» $\leftarrow$ vurpi ecc., żzurbignu e cal. (NDDC) survignu/so- «di sapore aspro» $\leftarrow \dot{z} z \dot{z}$ rbalsorva «sorba».

19. In realtà nel VS (III 826) è dato come "nome» piuttosto che come aggettivo. Ma, se così è, si tratterà di un deaggettivale a suff. $\varnothing$. VS = Vocabolario siciliano, [G. PICCITTO (a cura di) Vol. I (A-E); G. Tropea (a cura di) Vol. II (F-M); G. Tropea (a cura di), Vol. III $(N-Q)$; G. Tropea (a cura di), Vol. IV (R-Sgu); S.C. Trovato (a cura di), Vol V (Si-Z)], Catania-Palermo: Centro di Studi filologici e linguistici siciliani, 1977-2002.

20. Non è registrato nel VS, ma è vitale nel dialetto di Messina.

21. Si notino, per la semantica, le locc. ggenti di sgarru per "gente facinorosa ed equivoca» e mastru i sgarru "camorrista prepotente che bara al gioco» (VS IV 867). 
70 forme (incluse le varianti) in cui -ignu si lega a una base aggettivale, ed ha valore alterativo:

b) $\mathrm{A} \rightarrow \mathrm{A}$ :

acitignu, gacitignu "che tende all'acido» $\leftarrow$ (g)àcitu, aggualignul avalignulgualignu "pressoché uguale» $\leftarrow$ aggualilavalil(a)guali, agrignu "che ha un certo sapore agro" $\leftarrow$ agru, bbabbignu «mezzo scemo» $\leftarrow$ bbabbu, bbianchignulianchignu «biancastro» $\leftarrow$ bbianculiancu, bbiżzucchignu «alquanto bigotto» $\leftarrow$ bbiżzocca, cichignu "miope» $\leftarrow$ cecu, critinignu «mezzo scemo» $\leftarrow$ cretinu, crudignu «mezzo crudo" $\leftarrow$ crudu, ficilignu «selcioso, di terreno abbondante di silice» $\leftarrow$ ficila (in petra ficila "pietra focaia»), ${ }^{22}$ fuddignu «squilibrato; stravagante» $\leftarrow$ foddu e foddi, garrusignu «dinoccolato; lezioso, sdolcinato» $\leftarrow$ garrusu, ggialinignu "giallognolo" $\leftarrow$ ggiàlinu, ggiallignu "gialliccio» $\leftarrow$ ggiallu, ggirbignu/ggibbignu «di frutto non del tutto maturo» $\leftarrow$ ggerbu, lisignu «mezzo scemo" $\leftarrow$ lesu, lucchignu "che ha dello sciocco" $\leftarrow$ loccu «sciocco", mammalucchignu «inconcludente» $\leftarrow$ mammaluccu «sciocco", marrunignu "che dà sul marrone» $\leftarrow$ marroni, mbriachignu "mezzo ubriaco» $\leftarrow$ mbriacu, muntanignu «aquilino, del naso» $\leftarrow$ *muntanu, mutignu «mezzo muto, quasi muto» $\leftarrow$ mutu, muttignu «lento, fiacco» $\leftarrow$ mottu (con assimilazione, da mortu), nfastichignu "effeminato" $\leftarrow$ nfasticu "affettato, che veste con affettata eleganza», nivurignu «nericcio» $\leftarrow$ nivuru, parignu «della stessa pezzatura, di arance» $\leftarrow$ paru, pazzignu «squilibrato" $\leftarrow$ pazzula la paccigna "pazzescamente» $\leftarrow$ pàcciu "pazzo» (in area messinese), picchignu (lett. "pochigno») "persona rozza e di scarsa intelligenza» $23 \leftarrow$ picca "poco», picciriddignulpiccirillignu «di bassa statura e di corporatura esile» $\leftarrow$ picciriddu ecc., picciuttignu «dall'aspetto giovanile; che si comporta come un ragazzo» $\leftarrow$ picciottu, quartignulquattignu «di animale di quattro anni» $\leftarrow$ quartu, rrifuttutignu «astuto, scaltro» che presuppone un *rifuttutu, rrivirsignu «intrattabile, scontroso» $\leftarrow$ rriversu «id.», rrussignu/cal. russignu (NDDC) «rossiccio» $\leftarrow$ rrussu, ruçignu «dolciastro» $\leftarrow$ ruçi, rurignu «piuttosto duro" $\leftarrow$ ruru, saibbaggignu «che ha il sapore forte, tipico della selvaggina; dal carattere un po' selvatico' e savvaggignu «selvatico, di animale; timido, ritroso, di bambino" $\leftarrow$ saibbàggiu/savvàggiu, scimunitignu «mezzo scemo» $\leftarrow$ scimunitu, a la sciurbigna loc. avv. «alla cieca» $\leftarrow$ sciurbu «guercio, strabico", scurignu "che tende allo scuro, piuttosto scuro" $\leftarrow$ scuru, sfasciatignu "mezzo distrutto o logoro» $\leftarrow$ sfasciatu, sfausignu «alquanto sghembo» $\leftarrow$ sfausu, spirtignu «vivace, intelligente» $\leftarrow$ spertu, stupitignu «mezzo stupido» $\leftarrow$ stùpitu, stuttignu «un po» curvo, ecc.» $\leftarrow$ stottu (con assimilazione, da stortu), ${ }^{24}$ sulignu «solitario» $\leftarrow$ sulu, surdignu «sordastro» $\leftarrow$ surdu, tirzignultrizzignu «di animale di tre anni» $\leftarrow$ terzu, tisignu "teso" $\leftarrow$ tisu, turchinignu «turchiniccio, azzurrognolo» $\leftarrow$ turchinu, umitignu «umidiccio» $\leftarrow$ ùmitu, urbignu/ubbignulurvignu "dalla vista corta» $\leftarrow$ orbu, vappignu «mezzo guappo» $\leftarrow$ vappu, vicchjignu «rachitico» $\leftarrow$ vecchju, viccignu «che mostra

22. Nel cal. anche fucilignu (DEI II 1673). C. BatTisti, G. Alessio (et alii), Dizionario etimologico italiano, Firenze: Barbera 1950-57, (rist. anast 1968), 5 voll.

23. Anche m. «id.», ulteriore deaggettivale a suffisso zero.

24. Difficilmente il sic. sulignu può essere riportato all'it. solingo, come per i pochissimi casi di cui in (c), dal momento che non esiste nel sic. una forma del tipo sulinu. 
precoci segni di vecchiaia» $\leftarrow$ viècciu «vecchio» (in area ragusana), virdignu «verdiccio» $\leftarrow$ virdi, vivignu «assai vivace, sveglio» $\leftarrow$ vivu, zzaurdignu «di persona tozza, dai lineamenti poco fini» $\leftarrow$ zzaurdu, «urrignu «zotico, villano» $\leftarrow$ «urru «rozzo». 25

In altri casi -ignu è forma adattata di it. -ingo (sic. -ingu), ${ }^{26}$ e sicuramente prestiti dall'italiano sono:

c)

casalignu "casalingo» e guardignu (anche cal.) insieme a uardignulvaiddignul vardignu "guardingo». ${ }^{27}$ Vanno qui pure ricordati frisignu «esile» (accanto a frisingu), strutturalmente oscuro, sirignu «mingherlino» (accanto a siringu «esile snello", probabilmente da un ${ }^{*}(e)$ silingo).

Il nostro -ignu è forma parallela di -inu, altro suffisso derivativo del siciliano, in:

d)

murtaçignu «mogio» accanto a murtaçinu (lett. «morticino») e uminignu «maschile, di o da maschio» accanto al più diffuso umininu. La stessa cosa può dirsi del messin. trucchignu "che tende al turchino" per trucchinu (forma metatetica di turchinu) e di (campana) tucchigna ${ }^{28}$ "campano dal suono chiaro e squillante» (accanto a tucchinu «blu»).

Vanno, infine, ricordati:

$\left.\mathrm{d}_{1}\right)$

fuddarignu «matto, stravagante» accanto a fuddarinu e pazzarignu accanto a pazzarinu, con base, rispettivamente foddilfoddu "folle» e pazzu "pazzo» + suff. -arinu, che, assai probabilmente, proviene dalla risegmentazione di forme del tipo agghjar/ignu o agliarlinu "ghiaioso" ora interpretate come agghj/arinu e aglilarinu.

25. Oscuri, soprattutto per quel che riguarda la base, sono le seguenti altre forme in -ignu presenti nel VS: bbacchignu «stupido, babbeo», bbucignu «di bue giovane», fasilignu «mingherlino", rripitignu «di bassa statura» e zziffignu «cristallino, gelato». Mupignu «di chi non parla per omertà» è molto probabilmente un refuso per mutignu.

26. Peraltro estremamente raro nel sic., se col VS (V 70) si può aggiungere solo smilingu «esile, snello» accanto alle forme riportate in (c).

27. Particolare è sfacignu "guasto, deteriorato; mezzo sfatto, di frutta; malaticcio", che sembra postulare una base * sfaciri.

28. Impossibile, per motivi semantici, pensare a una base tuccu «turco». 


\section{Il suffisso -igno in D'Arrigo e Occhiato}

Il corpus derivante dallo spoglio esaustivo di $H O$ e di $O M$ è costituito da 11 parole provenienti da $H O$ e da 28 provenienti da $O M$. Di queste, sette sono comuni ai due romanzi ${ }^{29}$ e cioè: babbigno, ${ }^{30}$ citrigno, ${ }^{31}$ ferrigno, ${ }^{32}$ ladrigno, ${ }^{33}$ sanguigno, ${ }^{34}$ scaltrigno, ${ }^{35}$ e trucchigno $;{ }^{36}$ quattro sono esclusivi del romanzo darrighiano: caprigno, ${ }^{37}$ gialligno, ${ }^{38}$ rossigno ${ }^{39}$ e rossosanguigno, ${ }^{40}$ le rimanenti, ventuno, sono esclusive del romanzo di Giuseppe Occhiato. Si tratta di: cantarigno, ${ }^{41}$ cozzigno, ${ }^{42}$ dolcigno, ${ }^{43}$ drittigno, ${ }^{44}$ frichigno, ${ }^{45}$ gattigno, ${ }^{46}$ guardigno, ${ }^{47}$ lattigno, ${ }^{48}$ lupigno, ${ }^{49}$ mortigno, ${ }^{50}$ petrigno, ${ }^{51}$ piombigno, ${ }^{52}$ ramigno, ${ }^{33}$ serpigno, ${ }^{54}$

29. Non prendo in considerazione i diffusissimi benigno e maligno, ben presenti in entrambi i romanzi.

30. $H O$, p. 57, 204, 338, 486, 715, 912, 954, 9702 , 979, 1019; OM, p. 553. Si tenga presente che l'eventuale esponente del numero di pagina indica il numero di occorrenze all'interno della stessa pagina.

31. $H O$, p. $388,689,1239$; $O M$, p. $83,90,91,230,234,311,539,616,744,772,828,835$, $837,908,957,959,973,1033,1038,1060,1063,1068,1091,1099,1138,1186,1193$, 1229, 1287, 1299, 1305 .

32. $H O$, p. 55; OM, p. 54, 63, 125, 220, 247, 272, 283, 455, 474, 500, 707, 749, 829, 835, $846,859,967,1012,1046,1077,1098,1151,1270$.

33. $H O$, p. 54, 154; OM, p. 469, 704, 823 .

34. $H O$, p. 898, 944; $O M$, p. $154^{2}, 258,275,490,663,734,925,945$.

35. $H O$, p. 59, 411, 480, 578, 614, 625, 695, 715, 970, 979, 9812, 1019, 1102, 1103, 1176, 1196; OM, p. 24, 82, 110, 128, 131, 137, 336, 392, 433, 586, 5932 , 633, 636, 657, 695, 702, 706, 715, 719, 727, 731, 894, 945, 1004, 1148, 1152, 1212, 1240, 1242, 1249 , 1282.

36. $H O$, p. $16,149,197,302,317,382,589,659,668,799,968^{2}, 970,979,981 ; O M$ p. 26 , $31,78,195,219,224^{2}, 251,322,358,375,395,469,515,540,544,555,563,583,584$, $608,613,615,640,690,705,715,723,776,795,876,885,916,945,999,1029,1042$, $1057,1073,1132,1172,1181,1202,1232,1246,1258,1264,1287,1290,1293,1307$, 1321.

37. $H O$, p. 582,861 .

38. $H O$, p. $101,1082$.

39. $H O$, p. 222, 224, 235.

40. $H O$, p. 951.

41. $O M$, p. 1269.

42. $O M$, p. 77, 218, 234, 313, 525, 663, 747, 876, 945, 954, 1014, 1278.

43. $O M$, p. 98, 187, 408, 606, 612, 1027, 1203, 1222.

44. $O M$, p. $61,94,114,592$.

45. OM, p. 109, 181, 282, 520, 563, 596, 726, 900, 1298.

46. $O M$, p. $768,774,1236$.

47. $O M$, p. 769,859 .

48. $O M$, p. 23, 56, 61, 65, 82, 89, 111, 147,185, 259, 298, 335, 930, 946, 1114, 1288.

49. $O M$, p. 829,1165 .

50. $O M$, p. 10, 14, 26, 71, 117, 156, 218, 313, 358, 411, 417, 425, 453, 586, 607, 612, 773, 784, 794, 815, 855, 955, 968, 1006, 1041, 1061, 1103, 1110, 1141, 1157, 1185, 1232, 1298.

51. $O M$, p. $258,259,615,715,849,1289$.

52. OM, p. 130, 151, 152, 184, 281, 290, 317, 378, 677, 699, 1022, 1103, 1219, 1241, 1257.

53. $O M$, p. 614,704 .

54. $O M$, p. $193,532,597,716,720,794$. 
solfarigno, ${ }^{55}$ sordigno, ${ }^{56}$ surfarigno, ${ }^{57}$ survigno, ${ }^{58}$ vetrigno, ${ }^{59}$ volpigno ${ }^{60}$ e zolfi- $^{2}$ gno. ${ }^{61}$ In totale 32 parole in -igno, delle quali poco più di metà sono esclusive dei nostri due autori e non hanno trovato luogo nella lessicografia in lingua. Non si trovano, infatti, nei due maggiori repertori lessicografici italiani, il GDLI e il GRADIT:62 babbigno (HO e OM), cantarigno (solo OM), citrigno ( $H O$ e $O M$ ), cozzigno (solo $O M$ ), drittigno (solo $O M$ ), frichigno (solo OM), gattigno (solo OM), guardigno (solo OM), ladrigno (solo OM), lattigno (solo $O M)$, piombigno (solo $O M$ ), rossosanguigno (solo $H O$ ), scaltrigno (HO e $O M$ ), solfarigno (solo $O M$ ), sordigno (solo $O M$ ), surfarigno (solo $O M$ ), survigno (solo $O M)$ e trucchigno (HO e OM). Esse, pertanto, sono neoformazioni o perché attinte al siciliano o al calabrese e trasferite all'italiano letterario o perché create dai nostri due autori sulla base della creatività di -igno nelle loro parlate regionali.

Prima di presentare un breve scheda per quegli aggettivi assolutamente nuovi rispetto a quelli registrati nei dizionari italiani e dialettali, credo opportuno classificare gli usi letterari delle parole in -igno, distinguendo, ancora una volta, i denominali, i deaggettivali (alterati) e le altre forme in -igno che non rientrano tra le prime due categorie, secondo le eguenti categorie:

a) derivati:

$a_{1}$ ) denominali:

cantarigno $\leftarrow$ cantaro "misura di peso", caprigno $\leftarrow$ capra, citrigno $\leftarrow$ sic./cal. citru, cozzigno $\leftarrow$ cal. cozzu «nuca», ferrigno $\leftarrow$ ferro, gattigno $\leftarrow$ gatto, ladrigno $\leftarrow$ ladro, lattigno $\leftarrow$ latte, lupigno $\leftarrow$ lupo, petrigno $\leftarrow$ pietra, piombigno $\leftarrow$ piombo, ramigno $\leftarrow$ rame, sanguigno $\leftarrow$ sangue, serpigno $\leftarrow$ serpe, solfarigno $\leftarrow$ sòlfaro, surfarigno $\leftarrow$ cal. sùrfaru, survigno $\leftarrow$ cal. (e sic.) sorva "sorba", vetrigno $\leftarrow$ vetro, volpigno $\leftarrow$ volpe, zolfigno $\leftarrow$ zolfo;

$a_{2}$ ) deverbali:

frichigno $\leftarrow{ }^{*}$ fricare $<$ cal. fricari, guardigno $\leftarrow$ guardare;

55. $O M$, p. 376.

56. $O M$, p. $600,1374$.

57. $O M$, p. $251,606$.

58. $O M$, p. 250, 846, 1280.

59. $O M$, p. 1304.

60. $O M$, p. 529, 615, 756, 1204, 1297.

61. OM, p. 286, 689, 856, 965, 1247.

62. GDLI = S. BATTAGLIA, G. BARbERi SQuarotti (a cura di), Grande dizionario della lingua italiana, Torino: UTET, 1961-2002, [+ E. SANGUINETI (a cura di) Appendice, Torino: UTET, 2004; + G. RonCO (a cura di), Indice degli autori citati, Torino: UTET, 2004]. GRADIT = T. De MAUro, Grande dizionario italiano dell'uso, Torino: UTET, 1999-2003. 
b) alterati:

$\mathrm{b}_{1}$ ) a base aggettivale:

babbigno $\leftarrow$ babbo «stupido", dolcigno $\leftarrow$ dolce, drittigno $\leftarrow$ dritto, gialligno $\leftarrow$ giallo, mortigno $\leftarrow$ morto, rossigno $\leftarrow$ rosso, rossosanguigno $\leftarrow$ rossosangue, scaltrigno $\leftarrow$ scaltro, sordigno $\leftarrow$ sordo;

$\mathrm{b}_{2}$ ) a base nominale:

fratigno $\leftarrow$ frate per «fratello».

Segue ora l'elenco delle forme adoperate dai nostri due autori e di almeno un contesto d'uso, ${ }^{63}$ che non hanno ancora dignità lessicografica. Di ciascuna forma indico il modello dialettale quando l'origine di esse è sicuramente regionale e, nei casi in cui la base dialettale non è certa e si tratta di parola d'autore, fornisco l'analisi strutturale. Per quel che riguarda quest'ultima, si tenga presente che, quando la base non ha riscontro lessicale in italiano e in nessuno dei romanzi studiati, essa si configura, ovviamente, come confisso. ${ }^{64}$

BABBIGNO mezzo stupido, alquanto stupido

$$
\left[[\text { babbo }]_{\mathrm{N}}+\text {-igno }\right]_{\mathrm{A}}
$$

Non c'era cosa a mare di cui si potesse dire: quella tale cosa li spagna. ${ }^{65}$ C'erano però cose, che a farle, gli facevano senso. Come lanzare lo spada che può salvarsi la vita e viene a morire, impavido babbigno, per non staccarsi dalla coda della sua femmina, allora allora trafitta. (HO, p. 204);

Non sapeva, amaro cristiano, che quella era una sculimbra micidiale, calamitosa, che bastava sfiorarla, anzi guardarla una sola volta, rivolgerle la parola, farsi avvolgere dal suo sorriso per rimanerne travolto per sempre, per restarne come avvampato, martoriato di desiderio, pigliato in trappola senza rimedio, intrusciato e incascettato come il più babbigno dei baccalari, come il più locco dei matalocchi. (OM, p. 553).

CANTARIGNO agg. in salma cantarigna "carico molto pesante»

$$
\left[[\text { cantaro }]_{\mathrm{N}}+\text {-igno }\right]_{\mathrm{A}}
$$

Forse quella salma cantarigna che l'opprimeva, quell'orribile massa di spavento che lo soperchiava poteva rientrare nel mondo dei sogni, delle al|lucinazioni, poteva essere una figura evocata dalla sua immaginazione eccitata, stravolta (OM, p. 1269-70).

63. Viene solitamente scelto quello in cui la forma in -igno rappresenta meglio, semanticamente, il significato della base.

64. Cfr. per ciò Salvatore C. SGROI, «Per una ridefinizione di "confisso": composti confissati, derivati confissati, parasintetici confissati vs etimi ibridi e incongrui», Quaderni di Semantica, XXIV, 2003, p. 81-153 (p. 82-84).

65. E cioè «li spaventa». 
CITRIGNO dalla polpa soda, compatta, come quella del cedro $\left[[\text { citro }(<\text { sic./cal. citru })]_{\text {Conf. }}+{ }^{- \text {igno }}\right]_{\mathrm{A}} \quad(<$ sic., cal. citrignu $)$

Ma se l'erano rimirata più comodamente quando, come attirata dai loro sguardi, se n'era venuta a pipìare davanti al loro montarozzo: qui, ancora all'impie$\mathrm{di}$, si era tirata la veste sino ai reni, sino a scoprirgli in faccia la sua coffa di culo citrigno, poi si era abbassata e aveva fatto un grosso schizzo, scrosciante di schiuma nella rena. (HO, p. 686);

Sempre uguali a se stessi, quelli là, quelli come lui, tosti nella loro soperchiosità, arroganti e matamori perfino quando si trovava col culo per terra, ridotti alla desolazione dagli eventi di quelle giornate; e quale evento più citrigno per la loro bocca, più allarmante per la loro salvezza di quella cataratta che proprio in quei giorni stava sdilluvionando e ribellionando la Sigilia intera, che ormai si poteva dire caduta nelle mani dei nemici, [...] (OM, p. 539).

COZZIGNO ostinato, dalla dura cervice

$\left[[\operatorname{cozzo}(<\text { cal. cozzu })]_{\text {Conf. }}+{ }^{-i g n o}\right]_{\mathrm{A}}$

(< cal. cozzignu)

[I mali spiriti della notte] ancora non avevano lasciato il campo, come facevano ogni mattina ai primi lustri dell'alba, quando il canto dei galli faceva loro come di segnale e tutti scappavano a incaforchiarsi per tane| e dirupi; ancora facevano resistenza, cozzigni, cimentosi, restandosene rannicchiati sulle cornare invase dall'ombra, incufati nei vicoli oscuri, nei forni abbandonati, nei gafii deserti, o incastrati entro ai catoi dei sottoscala. $(O M$, p. 217-18).

DRITTIGNO «che sa o vuole essere furbo, scaltro, capace di ottenere ciò che vuole»

$$
\left[\left[\text { dritto }_{\mathrm{A}}+\text {-igno }\right]_{\mathrm{A}}\right.
$$

Dal modo come stava impostata, poggiata saldamente sui piedi semiaffondati nella rena, dimostrava di essere un vero esemplare del razzo femminino che regnava tra Scilla e Bagnara, dove le cristiane, sia marinare che cilonare, crescevano drittigne, passionali, più sperte e scaltrite degli stessi uomini, padri, fratelli, mariti e fidanzati; (OM, p. 61).

FRICHIGNO ingannatore

$\left[[\text { fricare }(<\text { cal. fricari })]_{\text {Conf. }}+\text {-igno }\right]_{\mathrm{A}}$

Che cosa gli stava capitando? Che cos'era che l'aveva trasformato, che l'aveva reso così mafrone, smaliziato, frichigno da mettersi sotto i piedi la coscienza, il dovere, l'onestà, fino a spregiare l'onore e il rispetto della zita? (OM, p. 109). 
GATTIGNO alla maniera dei gatti; simile ai gatti

$\left[[\text { gatto }]_{\mathrm{N}}+\text {-igno }\right]_{\mathrm{A}}$

Quanto mi piacete, Rizieri mio, mormoriava gattigna. Ah, quanto mi piacete! (OM, p. 768);

"Com'è che ti facesti pensierosa, che diventasti così taciturna? C'è qualcosa per cui sei triste?», attaccava, e la farfarella sollevava gli occhi gattigni a fissarlo. (OM, p. 774).

GUARDIGNO guardingo

$$
\left[[\text { guardare }]_{\mathrm{V}}+\text {-igno }(\text { per -ingo })\right]_{\mathrm{A}}
$$

Da quando il suo vecchio avversario aveva messo in giro quest'altra novità della sorella del minnatònnaro, si era fatto come più risoluto, guardigno, ma anche più spacenzioso, come se, levandosi una mattina con la zìrria d'Orlando, si ritrovasse che ce l'aveva con mezzo mondo. (OM, p. 769).

LADRIGNO ${ }^{66}$ da ladro, tipico del ladro

$\left[[\text { ladro }]_{\mathrm{N}}+- \text {-igno }\right]_{\mathrm{A}}$

[...] quella tredicina non si spagnava manco del gransatanasso in persona; era soltanto il nervino di non potersi pigliare la rivincita, ché attraverso la fessura delle palpebre strette, sotto i gigli disprezzosamente inarcati, dagli occhi ladrigni sprizzava lampi di ferocia, quasi volesse significargli: liberami, guercio cane, e fa' presto, ché poi ci penso io a levarmi la sgangata. (OM, p. 704).

LATTIGNO latteo, del colore del latte $\left[[\text { latte }]_{\mathrm{N}}+- \text {-igno }\right]_{\mathrm{A}}$

[Rizieri] arrivò alla riviera di Praialonga che baluginava nel suo iancore lattigno e quarzoso come una frangia fatta di seta, levigata e lucente, stretta fra la massa bituminosa del mare e la boscaglia della scarpata ferroviaria. (OM, p. 185).

PIOMBIGNO pesante come il piombo $\left[[\text { piombo }]_{\mathrm{N}}+\text {-igno }\right]_{\mathrm{A}}$

Dopo di questo, non fece più altri sogni ma continuò a dormire e dormì di un sonno piombigno fino a mezzogiorno, fino a quando cioè la zia Marangela non andò a risvegliarlo. (OM, p. 317).

66. Notevoli, anche, l'avv. Ladrignamente (p. 616, 732², 758, 823, 1053) e la loc. avv. alla ladrigna (p. 469). 
ROSSOSANGUIGNO di colore rossosangue

$\left[[\text { rossosangue }]_{\mathrm{A}}+\text {-igno }\right]_{\mathrm{A}}$

Sembrava però, che [...] non si fossero ancora minimamente scandaliati della presenza di un così nero, gigantesco, catastrofico animale, che avevano sotto occhio, lì, verso il paese delle Femmine, in quel mare stranamente colorato di rossosanguigno come fosse tempestato di corallo porporino, con qualcosa nel mezzo che aveva l'apparenza spaventosa di una colata di lava galleggiante. ( $H O$, p. 951).

SCALTRIGNO da scaltro, da furbo

$\left[[\text { scaltro }]_{\mathrm{A}}+\text {-igno }\right]_{\mathrm{A}}$

(nel sic. scattrignu)

Qui, di colpo, lasciò cadere quel tono smaccoso, quella mossa di voce iattante, spavalda, e tutto scaltrigno, diplomatico, si rivolse così a Nasomangiato: [...] (HO, p. 411).

«Guarda, guarda, appùrati se ce n’è una con la pinna soprana mozzata» gli fece suo padre, con un'aria che voleva essere scaltrigna e riusciva invece babbigna. (HO, p. 715).

[Rizieri] era stato per così lungo tempo lontano da lei e dal paese che si era lasciato facilmente affatare dalle tenerezze scaltrigne di quella fatamorgana, dalle carezze zingarelle di quella nimpia marinota. (OM, p. 24).

SOLFARIGNO infernale (che sa o puzza di zolfo)

$\left[\text { sòlfaro }_{\mathrm{N}}+\text {-igno }\right]_{\mathrm{A}}$

(<cal. surfarignu)

Quando rullavano i tamburi, specialmente se scordati, quando le potenti mazzole cantavano tabràn-tabràn-tabràn e la grancassa rispondeva col suo cuposo tum-tum-tùm, a stordimento, così, rapratrabranta-trabranta-trabranta, era cosa da non potersi soggiogare, peggio che al suono del corno fatato di Astolfo, e allora al babbionazzo malefico gli sarebbero saltati i denti dalle stesse mascelle, e alle bestiole [gli uccelli notturni e gli spiriti della notte] solfarigne sarebbe venuto il pisciasangue. (OM, p. 376).

SORDIGNO piuttosto cupo, smorzato (di suono)

$\left[\left[\right.\right.$ sordo $_{\mathrm{A}}+$-igno $_{\mathrm{A}}$

(nel sic. surdignu)

[...] i suonatori avevano attaccato un nuovo motivo, un ballabile così allegro e veloce, così cadenzoso che le loro mani e le loro dita si abbattevano come mazzole sulle corde ronzanti,| e i suoni erano alti, tumultuosi, stronanti come una ribellione di colpi sordigni, e la toccatocca delle mani e le scattagnette delle dita scandivano le note a tempo con la cadenza, [...] (OM, p. 600). 
SURFARIGNO v. solfarigno

$\left[[\text { sùrfaro }(<\text { cal. sùrfaru })]_{\text {Conf. }}+\text {-igno }\right]_{\mathrm{A}}$

E ancora una volta [don Sidorio] ebbe la rivelazione meravigliosa del segreto perduto di quel malessere surfarigno rinserrato nel nuovo regno ctonico delle nostre contrade da dove offriva la malanza e la spregiosità del proprio mistero. (OM, p. 251).

SURVIGNO aspro, piuttosto aspro (di sapore simile a quello delle sorbe) $\left[[\operatorname{sorva}(<\text { cal. sorva })]_{\text {Conf. }}+- \text { igno }\right]_{\mathrm{A}}$

(< cal. so-/survignu)

E qua| mio zio, che in certe questioni era cavilloso assai, per non dire sofistico, fu veramente zilloso, survigno, gli fece proprio male a don Sidorio Carrà, e pretese una spiegazione, [...] (OM, p. 249-50).

TRUCCHIGNO inaffidabile; alterato, fasullo

$\left[[\text { trucco }]_{\mathrm{N}}+\text {-igno }\right]_{\mathrm{A}}$

(nel cal. trucchignu)

Vecchiaia o rasoiate, quelle rughe o intacche, le stavano sulle guance come un tatuaggio con la data, di quando doveva essere assai sbardellata e invogliante, una gran campiona di galeota, passionale e tragediatora, e gli uomini che c'incappavano, o finivano in carcere o finivano al cimitero. Allo stato attuale, a lui, nell'insieme, gli riusciva sfrontata, trucchigna e scostante. (HO, p. 16).

Se l'artiglieria era antiquata, scarsa di potenza e insufficiente per numero di pezzi e per gittata, che dire di quelle sagome di grossicalibri trucchigni, capaci solo di sparare fumate, che dovevano servire a infasciare gl'ingenuazzi invasori $[\ldots](O M$, p. 26).

\section{Il suffisso -igno nella lessicografia recente e nelle grammatiche}

Riassumendo, il suffisso -ign|o in italiano serve a formare aggettivi di relazione quando la base è un nome (derivazione) e aggettivi alterati quando la base è un aggettivo. Casi come patrigno e matrigna potrebbero benissimo situarsi tra gli alterati nominali, avendo -igno, in questo caso, valore peggiorativo o attenuativo, ${ }^{67}$ mentre vitigno costituisce un caso a parte di derivazione $(\mathrm{N} \rightarrow \mathrm{N})$, con cambio di genere rispetto alla base.

Assolutamente oscuro, in sincronia, è privignol-a, essendosi perduta la trasparenza della base.

Sulla base di queste considerazioni e in rapporto alle funzioni che -ign|o di volta in volta assume nei processi ricordati, a livello lessicografico e diversamente dalla lessicografia recente che privilegia la polisemia sulla omonimia, propongo per -igno almeno due lemmi diversi:

67. Non è inutile richiamare, in questo caso, l'equivalenza tra it. -igno e sic. - ast $(r) u$ di cui sopra, $\$ 1$. 
-igno ${ }^{1}$, suff. che indica relazione, somiglianza, in aggettivi denominali;

$-i g n o^{2}$, suff. che, aggiunto ad aggettivi e a un ristretto numero di nomi che indicano relazioni di parentela (padre, madre), ha valore attenuativo.

I dizionari più recenti preferiscono la polisemia alla omonimia. Così il GRADIT (III 414) —il più attento sul piano formale - per il quale il suffisso igno «indica relazione, somiglianza in un ristretto numero di aggettivi denominali: benigno, caprigno, ferrigno, lupigno, volpigno» e, insieme «aggiunto ad aggettivi ha valore attenutativo: asprigno». Abbastanza preciso, in ordine alle funzioni del nostro suffisso, è lo Zingarelli $2008^{68}$ il quale, però, se da un lato dichiara esplicitamente i valori «derivativo» e «alterativo» del suffisso, nella parafrasi mescola insieme entrambe le funzioni di -igno: «forma aggettivi che esprimono attenuazione, approssimazione, somiglianza, talora con valore peggiorativo", e la stessa cosa fa nella esemplificazione, riportando in maniera indiscriminata i deaggettivali e i denominali: asprigno, dolcigno, ferrigno, rossigno, sanguigno, olivigno.

Per Sabatini-Coletti (e già prima anche sul DISC), infine, -igno è solo un "suffisso alterativo usato per formare approssimazione, somiglianza». ${ }^{69}$

Per quel che riguarda le grammatiche descrittive bisogna ricordare che Dardano Trifone correttamente annoverano -igno sia tra i suffissi che generano un aggettivo da un nome (ferrigno, sanguigno), sia tra gli alterati attenuativi. ${ }^{70}$ Serianni, invece, annovera -igno tra i suffissi con valore alterativo, ricordando che «in qualche caso, la base è un sostantivo: [...] ferro ferrigno, sangue sanguigno». ${ }^{71}$

Sul piano diacronico la situazione è un tantino più complessa. Intanto, -ign|o, suffisso aggettivale, rappresenta la convergenza dei latt. -INEUS e -IGNUS e, in taluni casi, è il risultato della risegmentazione di LARIG|NUS o SALIG|NUS, il cui /g/ fa parte della base, in lar|ignus o sal|ignus $;{ }^{72}$ in altri casi — come ad es. patrigno, matrigna e il recente fratigno di Giuseppe Occhiato- l'ign|o, come si è già visto, è il risultato della grammaticalizzazione della radice * gen, originariamente presente in PRIVIGNUS/-A (> privigno/-a) da cui si è estesa, già in latino, a matrigna e quindi a patrigno (v. supra $\$ 1$ ) e che non è difficile ravvisare in benigno, ciprigno, maligno e vitigno.

Il suffisso per Rohlfs è assai usato nel Meridione. A questo proposito lo studioso ricorda il salent. nustersignu «avanti ieri l'altro», con -ignu con valore

68. Zingarelli $2008=$ N. Zingarelli, Vocabolario della lingua italiana, Bologna: Zanichelli, 2007.

69. F. Sabatini, V. Coletti, Il Sabatini Coletti. Dizionario della lingua italiana 2004, Milano: Rizzoli Larousse, 2003. DISC= Dizionario italiano Sabatini Coletti, Firenze: Giunti 1977.

70. M. DaRdano; P. TRIFOne, La nuova grammatica della lingua italiana, Bologna: Zanichelli, 1997, p. 531, 540.; M. DARDANO; P. TRIFONE, Grammatica italiana con nozioni di linguistica, Bologna: Zanichelli, 2007³, p. 597, 606.

71. L. SerianNI, Grammatica italiana. Italiano comune e lingua letteraria. Suoni Forme Costrutti (con la collaborazione di A. CASTELVECCHI), Torino: UTET, 1988, p. 546.

72. P. TeKavČIĆ, Grammatica storica dell'italiano, Vol. III: Lessico, Bologna: Il Mulino 1972, p. 105; G. RoHLFS, Grammatica storia della lingua italiana e dei suoi dialetti. Sintassi e formazione delle parole, Torino: Einaudi, 1969, p. 389. 
diminutivo e base avverbiale, che possiamo confrontare, per il valore alterativo, col sic. avantirazzu «alcuni giorni prima dell'altro ieri». ${ }^{73}$

\section{Bibliografia}

DEI = Battisti, C.; Alessio, G. (et alii), Dizionario etimologico italiano, Firenze: Barbera 1950-57, rist. anast 1968, 5 voll.

Disc = DISC. Dizionario italiano Sabatini Coletti, Firenze: Giunti 1977, con CDRom

$\mathrm{DM}=\mathrm{De}$ Mauro, T. a cura di, Il dizionario della lingua italiana, Torino: Paravia 2000, con CD-Rom.

GDLI = BATTAGLia, S.; BARBERI SQUarotTI, G. a cura di, Grande dizionario della lingua italiana, Torino, UTET 1961-2002, voll. I-XXI, + Appendice a cura di E. SANGUINETI, ibid. 2004 + Indice degli autori citati, a cura di G. RONCO, ibid. 2004.

GRADIT = Grande dizionario italiano dell'uso, ideato e diretto da T. DE MAURO, 7 voll. Torino: Utet 1999-2003, con CD-Rom.

Grossmann, M.; Rainer, F. a cura di, La formazione delle parole in italiano, Tübingen: Niemayer 2004.

MERLINI BARBARESI, L. Alterazione, in Grossmann-Rainer, p. 264-292.

NDDC = RohlfS, G. Nuovo Dizionario dialettale della Calabria, Ravenna: Longo 1977.

Piromalli, A. Giuseppe Occhiato narratore epico-popolare, in «Letteratura \& Società», anno IV, n. 2, maggio-agosto 2002, fascicolo 11, p. 35-50.

RoHLFs, G. Grammatica storia della lingua italiana e dei suoi dialetti. Sintassi e formazione delle parole, Torino: Einaudi 1969.

Sabatini-Coletti = SABATINI, F.; ColetTI, V. Il Sabatini Coletti. Dizionario della lingua italiana 2004, Milano: Rizzoli Larousse 2003, con CD-Rom.

SCALISE, S. La formazione delle parole, in Grande grammatica italiana di consultazio$n e$, a cura di L. RENZI et alii, Bologna: Il Mulino $1995^{1}, 2001^{2}$, vol. III, cap. 10, p. 471-516; (bibl.) p. 578-87.

SGROI, S. C. (Im)produttività dei suffissi -oso, -ioso, -uoso e problemi di etimologia sincronica e diacronica, in "Quaderni di Semantica», a. XXI, 2000, p. 265-319.

SGROI, S. C. Per una ridefinizione di "confisso": composti confissati, derivati confissati, parasintetici confissati vs etimi ibridi e incongrui, in "Quaderni di Semantica», a. XXIV, 2003, p. 81-153.

Trovato, S. C. La formazione della parole in Horcynus Orca di Stefano D'Arrigo. Tra regionalità e creatività, in "Quaderni di Semantica», a. XXVIII, 2007, p. 41-88.

Trovato, S. C.; VAlenti, I. (in stampa), Vocabolario siciliano inverso.

VS = Vocabolario siciliano, vol. I (A-E) a cura di G. PICCITTO, Catania-Palermo 1977; vol. II (F-M) a cura di G. TROPEA, Catania-Palermo 1985; vol. III (N-Q), a cura di Giovanni TropeA, Catania-Palermo 1990; vol. IV (R-Sgu) a cura di G. TropeA, Catania-Palermo 1997; vol V (Si-Z) a cura di S. C. Trovato, Catania-Palermo 2002, Centro di Studi filologici e linguistici siciliani.

WANDRUSZKA, U. Aggettivi denominali, in Grossmann-Rainer p. 382-402.

Zingarelli, N. (2008). Vocabolario della lingua italiana, Bologna: Zanichelli 2007. 